Scientific Journal of Hamadan Nursing \& Midwifery Faculty - ISSN 2008-2819

\title{
The Relationship Between Sleep Quality with Cognitive Emotion Regulation Strategies and Brain-Behavioral Systems in Pregnant Women
}

\author{
Jahangir Karami ${ }^{1}$, Khodamorad Momeni ${ }^{1}$, Faride Alimoradi ${ }^{2 *}$
}

1. Associate Professor, Department of General Psychology, Faculty of Social Sciences, Razi University, Kermanshah, Iran

2. MSc., Department of General Psychology, Faculty of Social Sciences, Razi University, Kermanshah, Iran

\begin{tabular}{ll}
\hline \multicolumn{2}{c}{ Article Info } \\
\hline & \\
Received: & $2017 / 07 / 16$ \\
Accepted: & $2017 / 09 / 24$ \\
Published Online & $2018 / 03 / 16$
\end{tabular}

DOI:

10.30699/sjhnmf.26.4.219

Original Article

Use your device to scan and read the article online

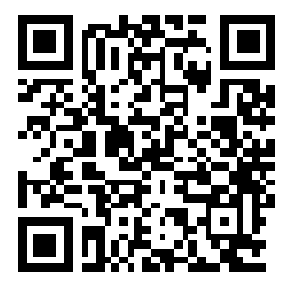

\section{Abstract}

Introduction: Reduction of the quality of sleep is one of the most common complaints of women during pregnancy that leads to negative consequences on the mental health of pregnant women. Some factors that associate with quality of sleep are how to use cognitive emotion regulation strategies and brain-behavioral systems. This investigation aims to study the relationship between sleep quality with cognitive emotion regulation strategies and brain-behavioral systems in pregnant women.

Methods: This study was conducted in the first half of 2016 on 302 pregnant women who referred to health centers in Kermanshah city and were chosen using cluster sampling method. Subjects completed the Pittsburgh sleep quality questionnaire, Garnefski cognitive emotion regulation strategies questionnaire and Carver and White BIS/BAS questionnaire with a self- assessment method. Data was analyzed using descriptive statistical, Pearson's correlation and regression and statistical and SPSS22. The significant level considered as: $(P<0 / 05)$.

Results: There was significant relationship between sleep quality with emotion regulation strategies and behavioral inhibition system; the use of negative and maladaptive cognitive emotion regulation strategies and high activity in the behavioral inhibition, decrease the quality of sleep in pregnant women.

Conclusion: Using some of the negative and maladaptive cognitive emotion regulation strategies such as rumination and disaster concept, and high activity in behavioral inhibition system can decrease sleep quality in pregnant women.

Keywords: Pregnancy, Cognitive emotion regulation strategies, Brain-Behavioral systems, Sleep quality 


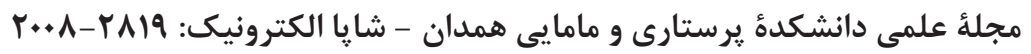

مقالأ يزوهشى

بررسى رابطؤ كيفيت خواب با راهبردهاى تنظيم شناختى هيجان و سيستمهاى مغزى ـ رفتارى در زنان باردار

جهانگير كر مى'، خدامراد مؤمنى'، فريده عليمر ادى"”

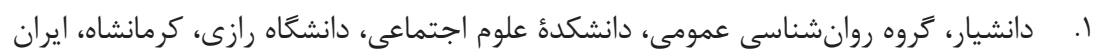

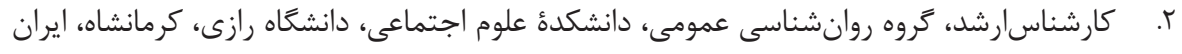

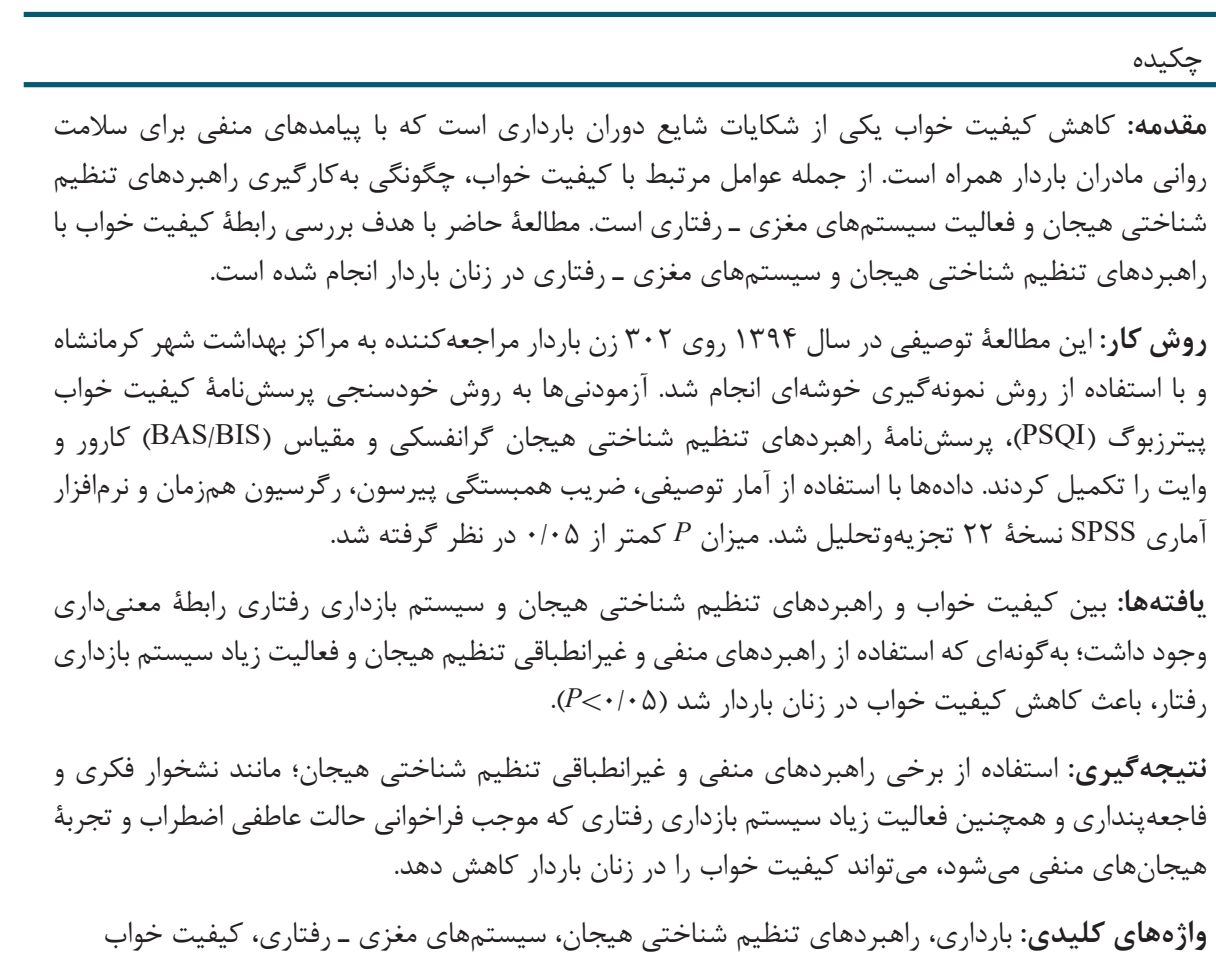

مقلدمه

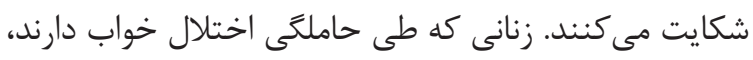

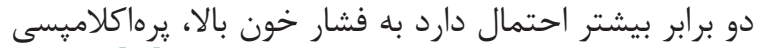

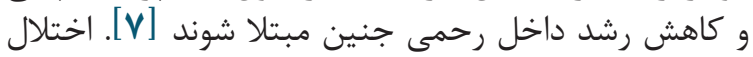

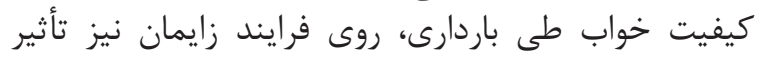

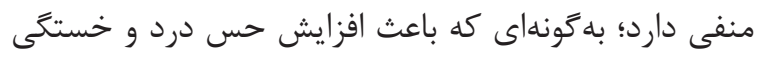

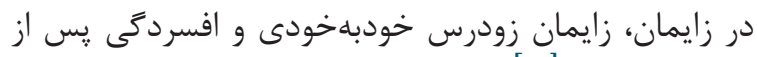
زايمان مىشود [1/.

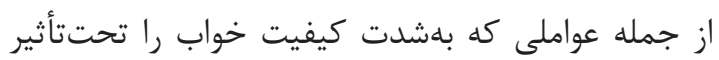

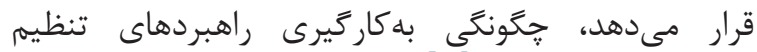

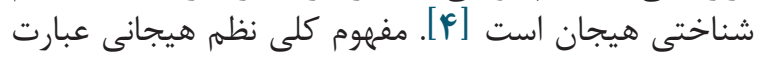

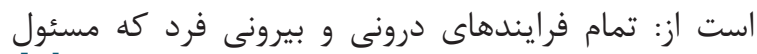

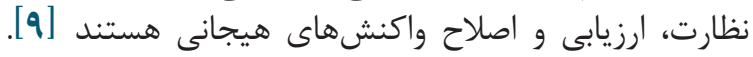

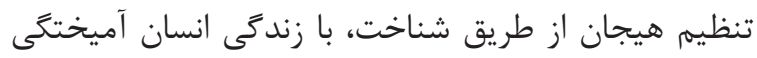

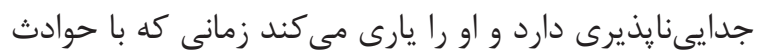

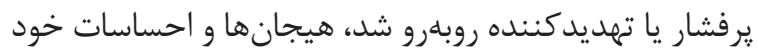

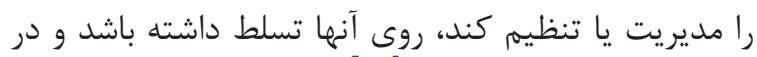

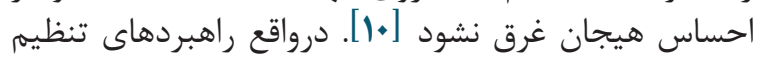

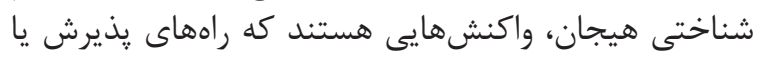

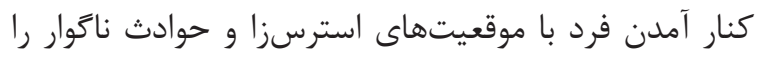

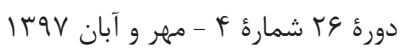

\begin{tabular}{|c|c|}
\hline \multicolumn{2}{|c|}{ اطلاعات مقاله } \\
\hline $\mid r q s / 4 / Y \Delta$ & تاريخ وصول: \\
\hline Irq9/N/T & تاريخ یذيرش: \\
\hline$|r q 9 /| r / T \Delta$ & انتشار آنلاين: \\
\hline & نويسندهُ مسئول: \\
\hline & فريده عليمر ادى \\
\hline وانشناسى & كارشناسارشد، گرو \\
\hline اجتماعى، & عمومى، دانشكدة \\
\hline ش شاه، ايران & دانشعاه رازى، كرما \\
\hline
\end{tabular}

קֶست الكترونيك:

faridealimoradi.zendegi@gmail.com

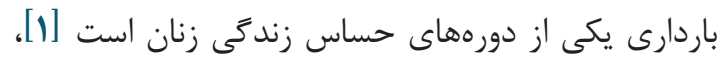

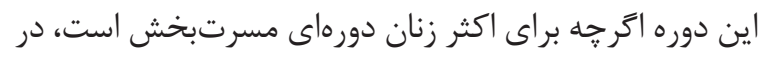

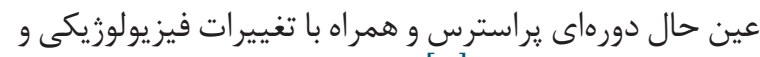

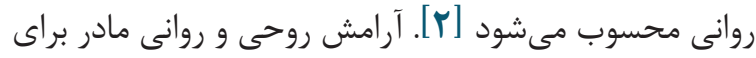

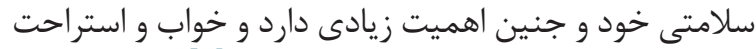

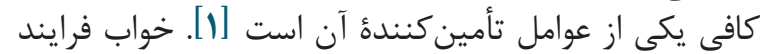

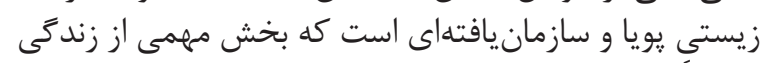

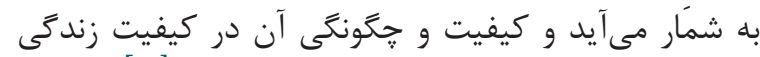

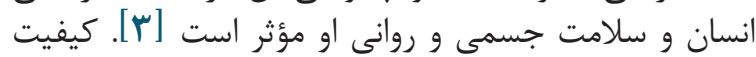

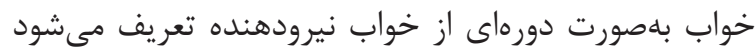

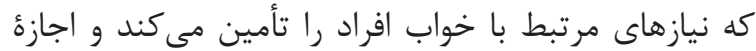

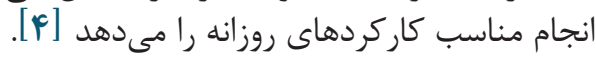

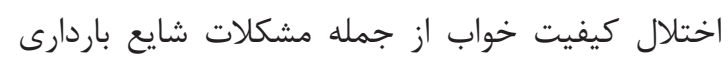

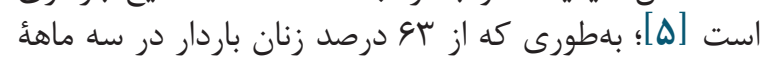

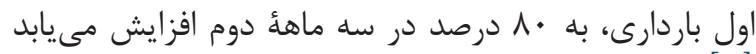

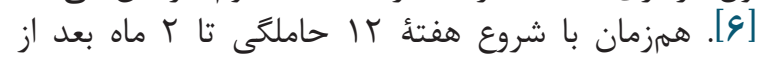

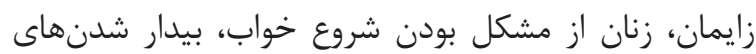

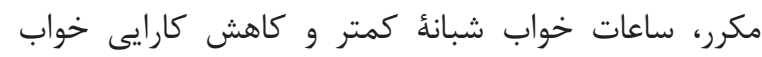

$$
\text { مجله علمى دانشكدة يرستارى و مامايى همدان }
$$




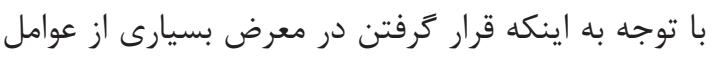

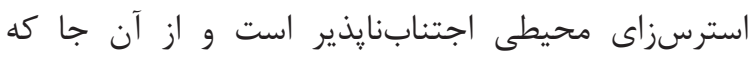

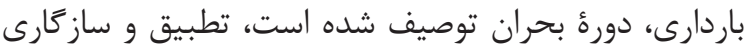

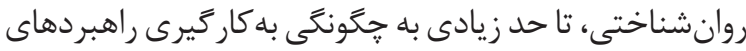

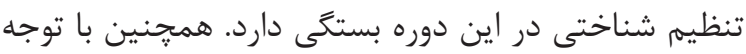

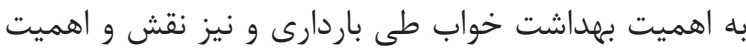

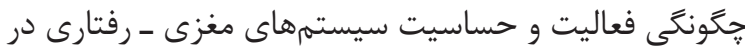

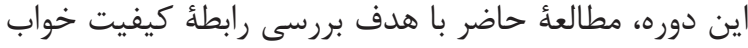

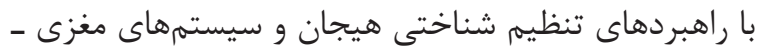

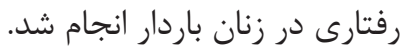

\section{روش كار}

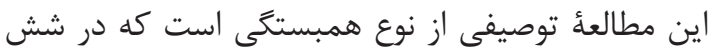

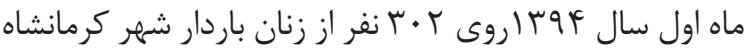

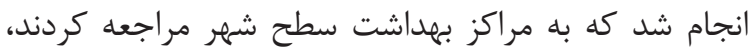

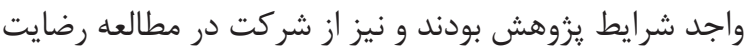

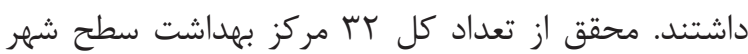

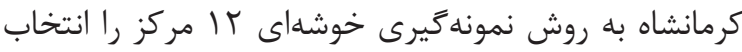

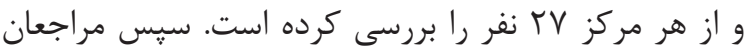

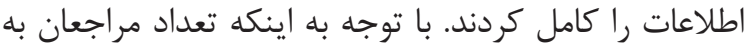

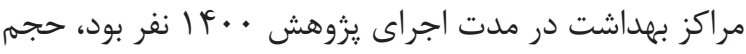

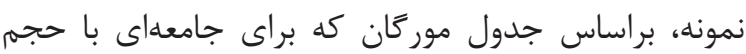

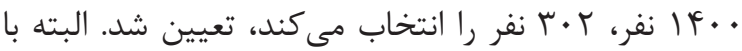

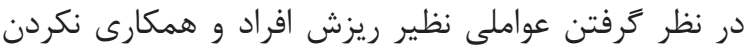

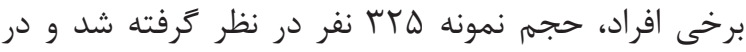

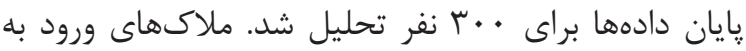

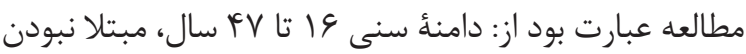

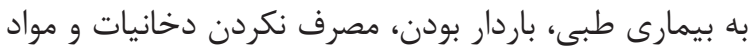

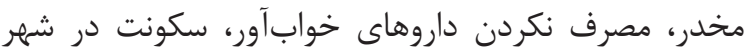

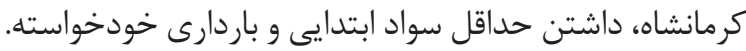

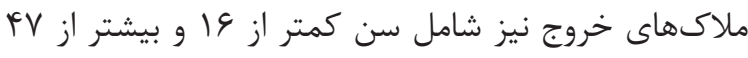

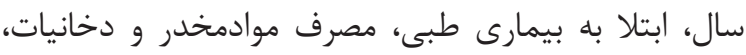

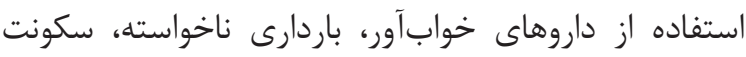

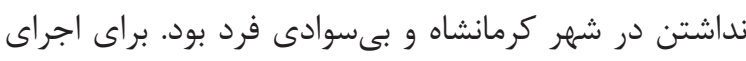

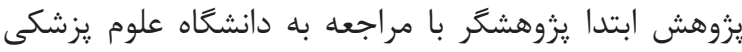

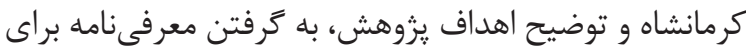

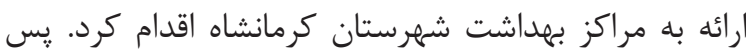

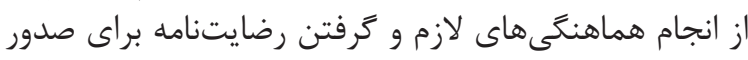

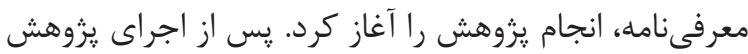

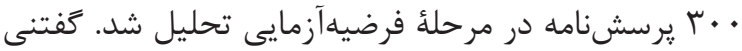

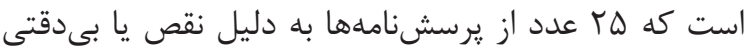

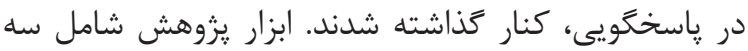

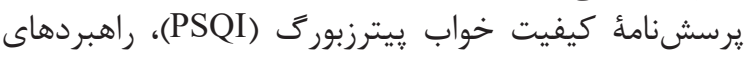

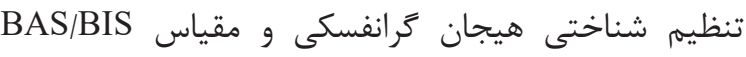
كارور و وايت بود. ت كئن

\section{الف-يرسشنامهٔ كيفيت خواب پيترزبورى (PSQI):}

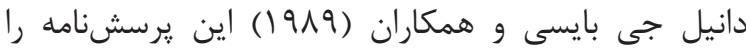

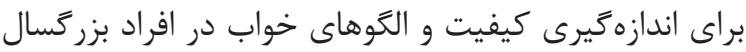

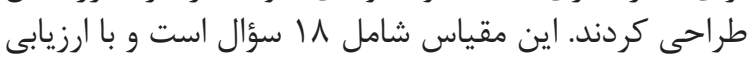

معين مى كنند [1/]. اين راهبردها به دو دستأ مثبت و منفى تقسيم مىشوند [1/]].

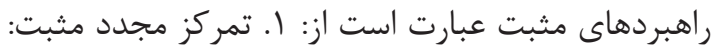

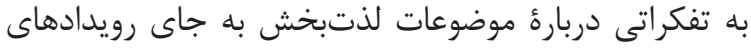

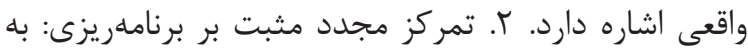

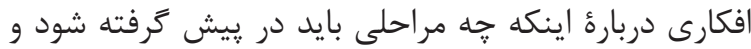

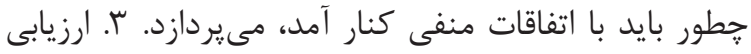

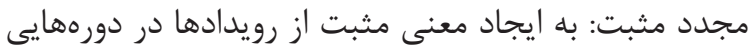

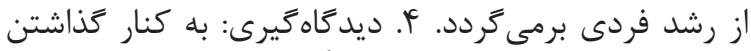

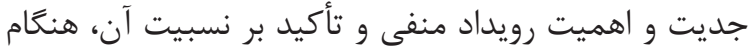
مقايسه با ديكر رويدادها اطلاق مىشود.

راهبردهاى منفى عبارت است ازي: إ. سرزنش خون خودي:

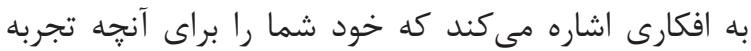

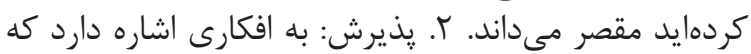

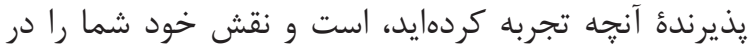

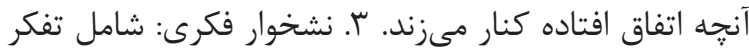

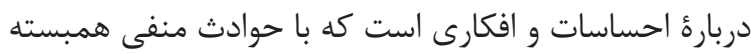

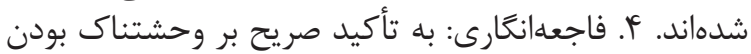

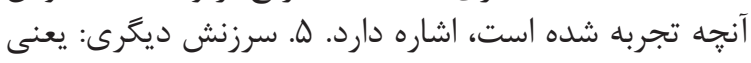

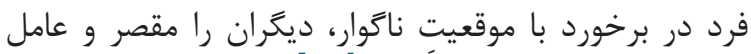

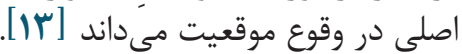

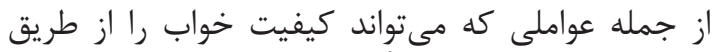

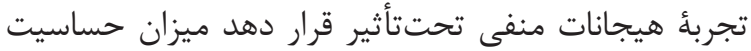

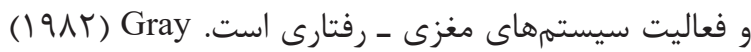

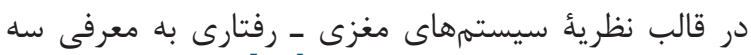

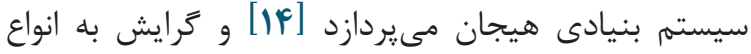

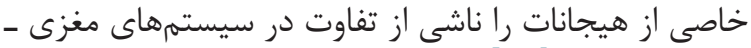

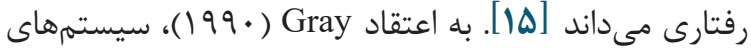

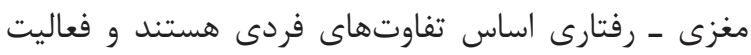

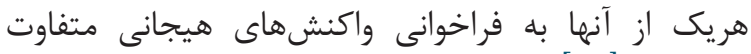

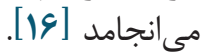

طبق نظرئ Gray اولين سيسته، سيستم فعال ساز رفتارى (BAS)

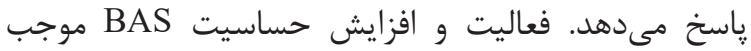
فراخوانى هيجانهاى مثبت، رفتار فيتار روى آورى، اجتناب فيت فعال

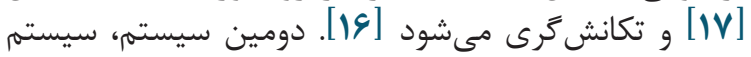

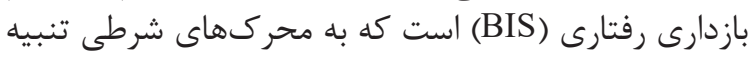

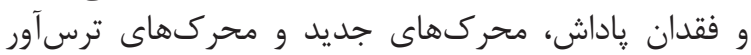

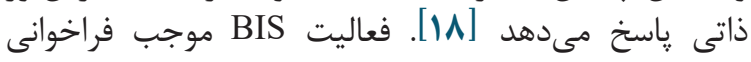

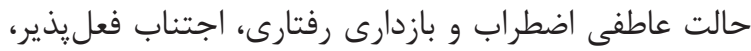

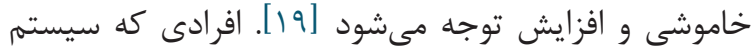

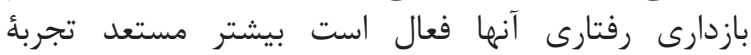

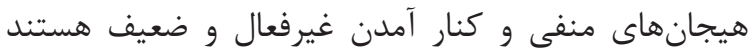

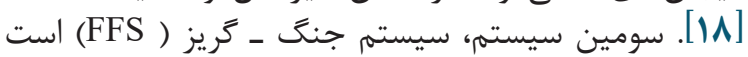

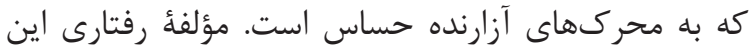

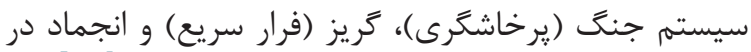

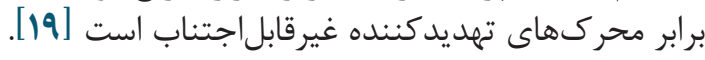


تجزيلوتحليل شد. توزيع جامعه (نرمال بودن توزيع دادهها)

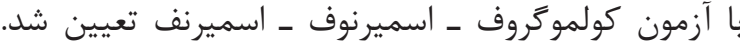

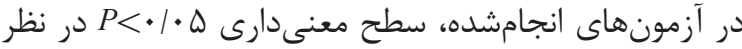

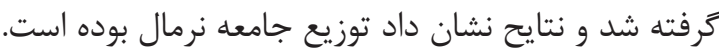

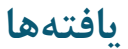

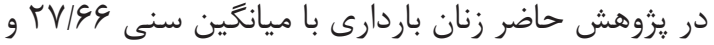

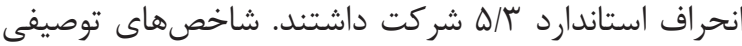

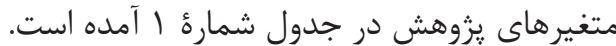
ميانكين و انحراف استاندارد متغييرهاى كيفيت خواب، تبني

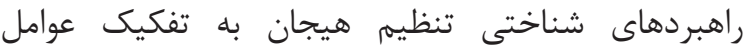

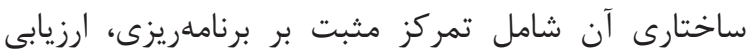

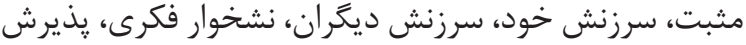

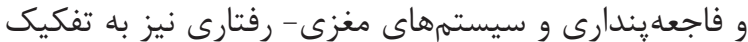

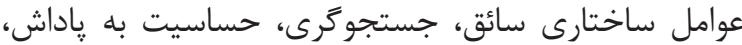

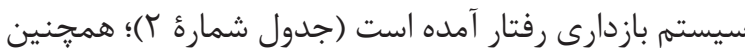

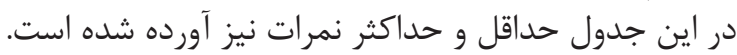
ضرايب همبستگى بين راهبردهاى شناختى تنظيم

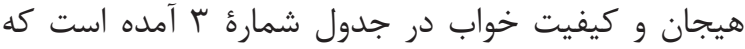

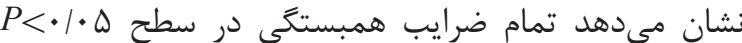

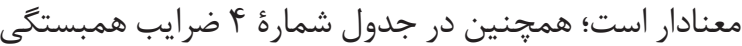

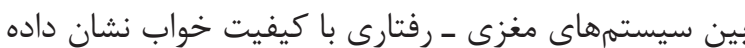

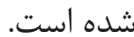

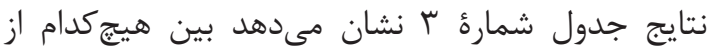

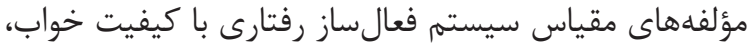

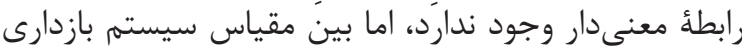

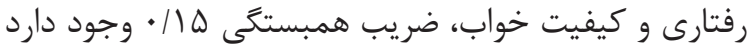

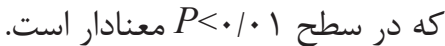

براى ييشبينى كيفيت خواب براساس راهبردهاى تنظيم

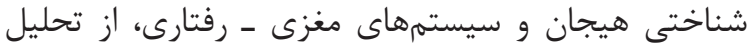

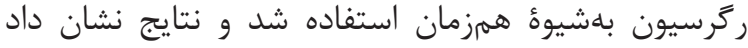

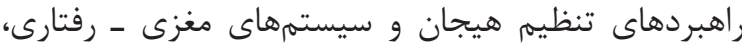

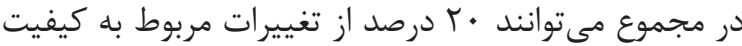
خواب را ي بيشبينى كنند.

در جدول شماره ه ضرايب پِيشبينى كيفيت خواب

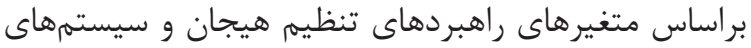
مغزى ـ رفتارى، طبق آزمون تحليل ركرسيون هيجان آمده است. نتايج تحليل ركرسيون كه در جدول شماره هـ آمده است،

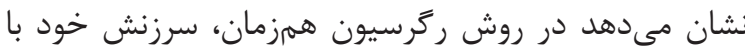

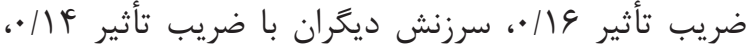

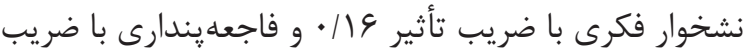

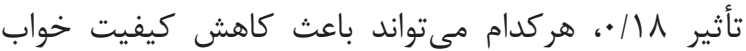

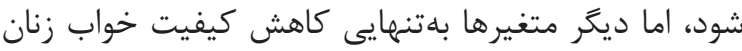

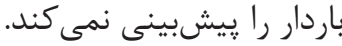

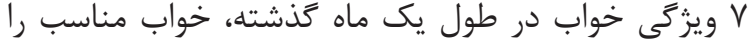

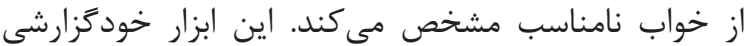

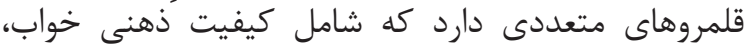

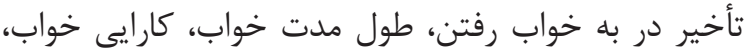

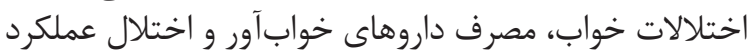

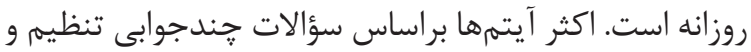

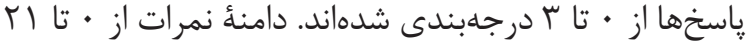

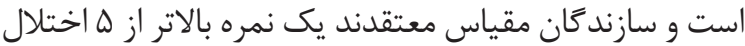

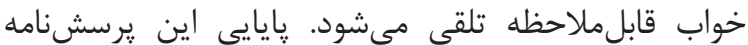

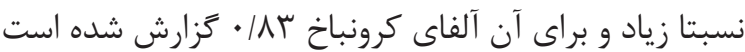

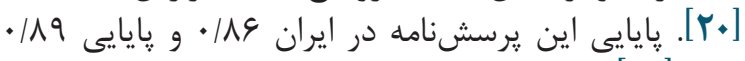

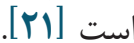

ب- برسشنامهُ تنظيم شناختى هيجان: در سال

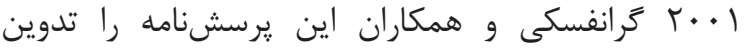

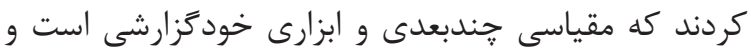

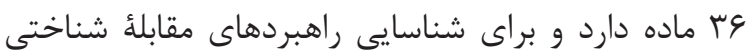

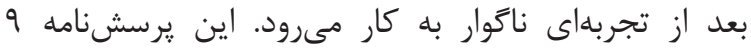

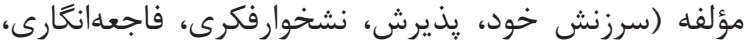

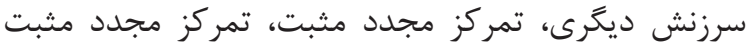

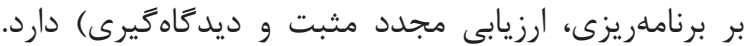

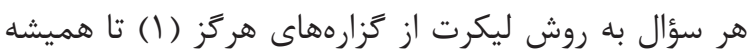

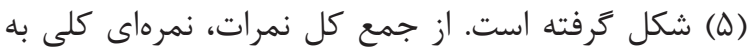

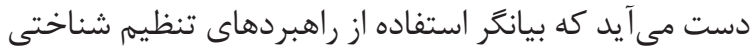

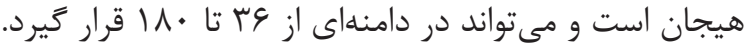

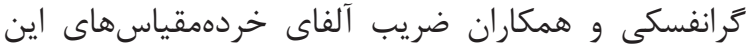

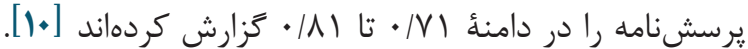

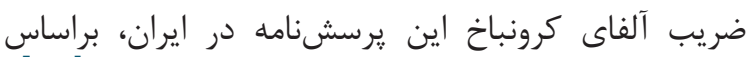

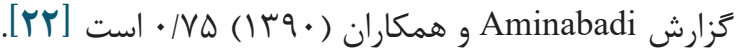

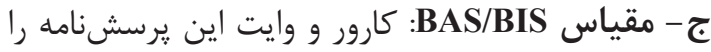
در سال جوال مقياس براى ارزيابى حساسيت سرشتى

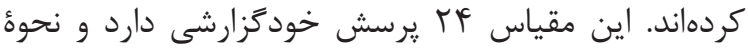

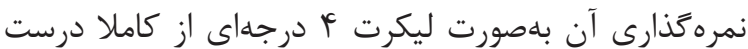

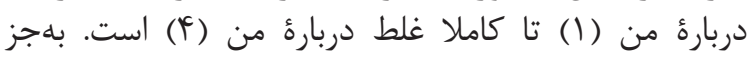

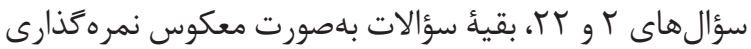

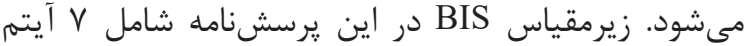

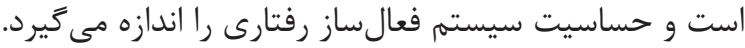

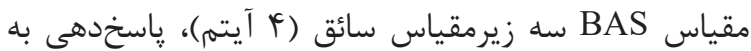

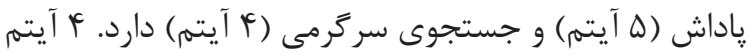

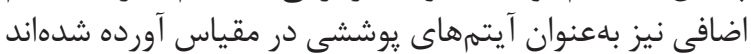

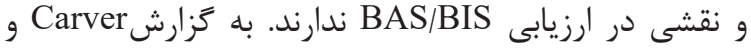

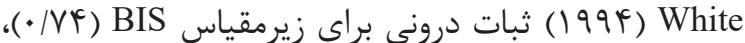

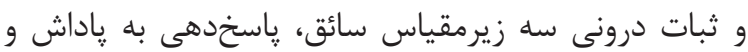

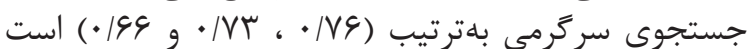

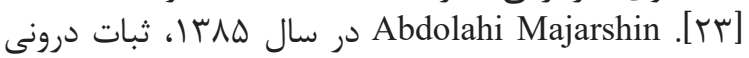

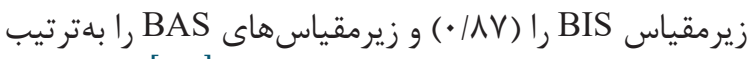
)

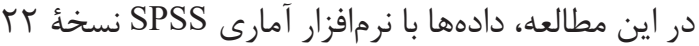

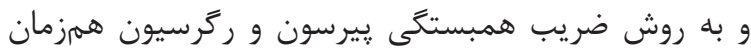


جدول شماره ا. شاخصهاى توصيفى متغيرهاى يزوهش

\begin{tabular}{|c|c|c|c|}
\hline درصد & فراوانى & & متغير \\
\hline$r \& / r$ & vq & زيردييلم & \multirow{3}{*}{ وضعيت تحصيلى } \\
\hline 每 & $1 \cdot 1$ & دييلهم & \\
\hline$r V / V$ & $11 \pi$ & بالاى دييله & \\
\hline$\cdot \mid \Delta \varphi$ & 191 & اول & \multirow{3}{*}{ زايمان } \\
\hline$\cdot / r V$ & $11 \pi$ & دوم & \\
\hline$\cdot / V$ & 19 & سوم و بيشتر & \\
\hline$f 1 / 9$ & IrG & ماه اول تا پنجمم & \multirow{2}{*}{ ماه باردارى } \\
\hline$\cdot \mid \Delta \Lambda$ & IVF & ماه ششم تا نهمم & \\
\hline$I V / V$ & $\Delta r$ & با سابقه سقط & \multirow{2}{*}{ سقط } \\
\hline$\Lambda T / r$ & TFV & بدون سابقه سقط & \\
\hline
\end{tabular}

جدول شمارء ז. ميانَّين و انحراف استاندارد متغييرهاى كيفيت خواب، راهبردهاى شناختى تنظيم هيجان و سيستمهاى مغزى - رفتارى

\begin{tabular}{|c|c|c|c|c|c|}
\hline انحر اف استاندارد & ميانگين & 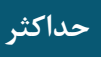 & حداقل - & & \\
\hline s/Vq & $\mu F / \cdot \Lambda$ & QI & 10 & كيفيت خواب & كيفيت خواب \\
\hline$\Delta / 9$. & $r V / T V$ & $i \Delta$ & 11 & تمركز مثبت بر برنامهريزى & \multirow{7}{*}{ راهبردهاى شناختى تنظيم } \\
\hline$f / T V$ & $19 / \mu r$ & $r \cdot$ & 4 & ارزيابى مثبت & \\
\hline$r / 19$ & $9 / 4 \varepsilon$ & if & f & سرزنش خود & \\
\hline$r / \Delta 9$ & N/FF & 10 & r & سرزنش ديخران & \\
\hline$T / \Delta F$ & $\mid F / \Delta V$ & TY & $\Delta$ & نشخوار فكرى & \\
\hline$r / 1 Q$ & $\mid r / \Delta \varphi$ & $r$. & \& & يذيرش & \\
\hline$r / \cdot r$ & $\mid r / \Delta \Lambda$ & $r \cdot$ & r & فاجعهيندارى & \\
\hline$r / T q$ & 1.194 & 19 & f & سائق & \multirow{4}{*}{ سيستمهاى مغزى - رفتارى } \\
\hline$T / / Q$ & $11 / T \Delta$ & 19 & r & جستجوكرى & \\
\hline 1/99 & IV/VT & $r \cdot$ & 11 & حساسيت به پاداش & \\
\hline$r|9|$ & $r \cdot / \Lambda r$ & TV & if & سيستم بازدارى رفتار & \\
\hline
\end{tabular}

جدول شمارؤ س. ضرايب همبستخى بين راهبردهاى شناختى تنظيم هيجان و كيفيت خواب

\begin{tabular}{|c|c|c|}
\hline سطح معنادارى & ضرايب همبستَى & راهبردهاى تنظيم هيجان \\
\hline$\cdot 1 \cdot \cdot 1$ & $-\cdot / r q$ & تمركز مثبت بر برنامهريزى \\
\hline$\cdot 1 \cdot \cdot 1$ & $-\cdot / T F$ & 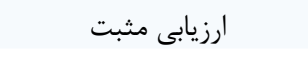 \\
\hline$\cdot 1 \cdot \cdot 1$ & $\cdot / \mu F$ & سرزنش خود \\
\hline$\cdot / \cdot \cdot 1$ & $\cdot / 49$ & سرزنش ديكران \\
\hline$\cdot 1 \cdot \cdot 1$ & $\cdot / \pi \Delta$ & 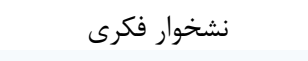 \\
\hline$\cdot 1 \cdot \cdot 1$ & $\cdot / 49$ & 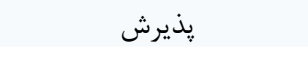 \\
\hline$\cdot 1 \cdot \cdot 1$ & $\cdot \pi \cdot$ & فاجعه يندارى \\
\hline
\end{tabular}


جدول شمارة F. ضرايب همبستخى بين سيستمهاى مغزى ــ رفتارى با كيفيت خواب

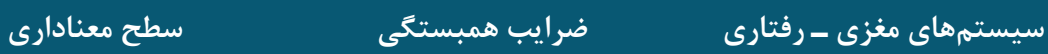

\begin{tabular}{|c|c|c|}
\hline.$/ 99$ & $\cdot / \cdot 1$ & سائق \\
\hline$\cdot / T \Delta$ & $\cdot / \cdot V$ & جستجوگرى \\
\hline$\cdot / 9$. & $\cdot / \cdot V$ & حساسيت به پاداش \\
\hline$\cdot / \cdot 1$ & .110 & سيستم بازدارى رفتارى \\
\hline
\end{tabular}

جدول شماره ه. ضرايب ركر سيونى براى ييشبينى كيفيت خواب

\begin{tabular}{|c|c|c|c|c|}
\hline معنادارى & $\mathbf{t}$ & $\boldsymbol{\beta}$ & B & متغير بيش بين \\
\hline$\cdot / \mu F$ & $\cdot 190$ & $\cdot / \cdot \Delta$ & $\cdot / 1 F$ & سيستم بازدارى رفتارى \\
\hline$\cdot 19$ & $\cdot / \Delta T$ & $-\cdot / \cdot f$ & $-\cdot / \cdot \Delta$ & تمركز مثبت بر برنامهريزى \\
\hline$\cdot / f F$ & $\cdot / V \wedge$ & -.1 .9 &.$- / 1$ & ارزيابى مثبت \\
\hline$\cdot 1 \cdot 1$ & T/AF & .119 & $\cdot / \Delta T$ & سرزنش خود \\
\hline$\cdot 1 \cdot r$ & $r / T V$ &.$/ 1 F$ & $\cdot r v$ & سرزنش ديخران \\
\hline$\cdot 1 \cdot r$ & $r / \cdot \Delta$ & .119 & $\cdot \pi \cdot$ & نشخوار فكرى \\
\hline$\cdot / 49$ & $\cdot / V F$ & $\cdot 1 \cdot \Delta$ & .111 & يذيرش \\
\hline$\cdot / \cdot v$ & T/VT &.$/ 11$ & $\cdot|4|$ & فاجعهيندارى \\
\hline
\end{tabular}

ناخوار مقصر مىدانند و به سرزنش خود مىيردازند و

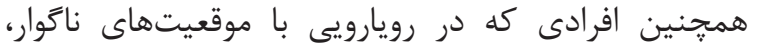

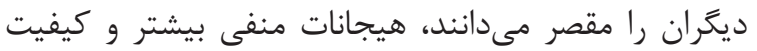

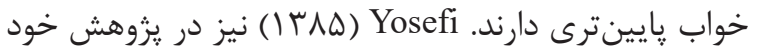

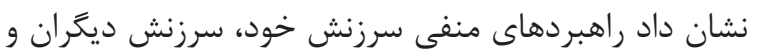

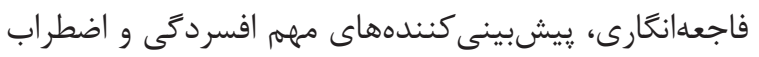

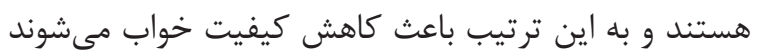

[rA]

در مقابل، بين استفاده از راهبردهاى مثبت تنظيمى شناختى

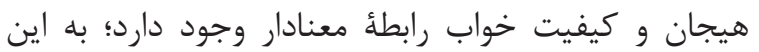

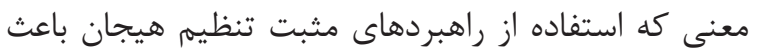

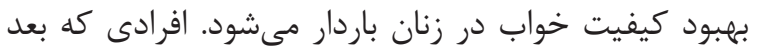

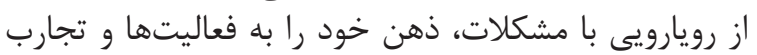

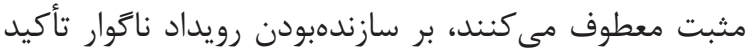

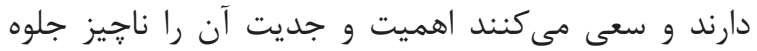

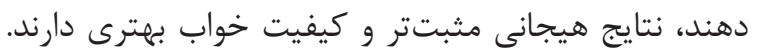

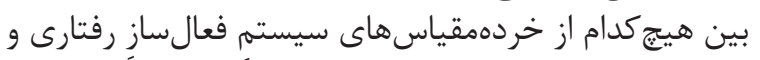

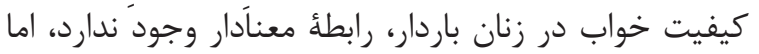

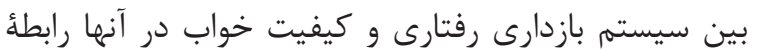

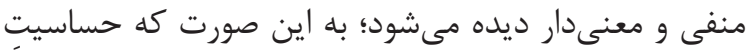

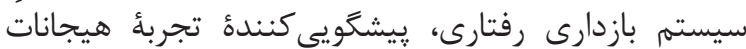

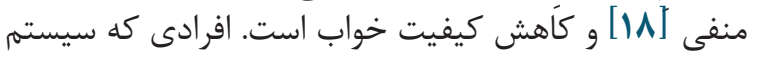

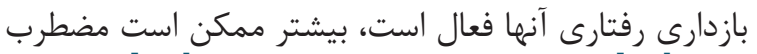

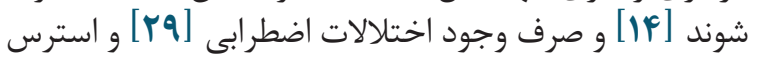

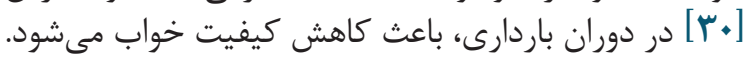

بحث در اين مطالعه بين تمام مؤلفههاى منفى و غيرانطباقى

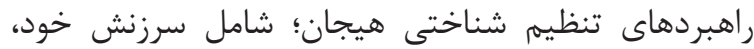

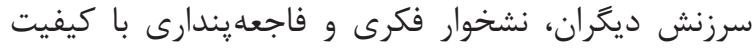

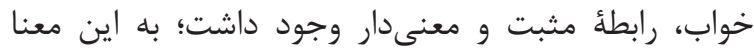

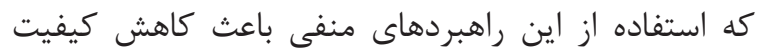

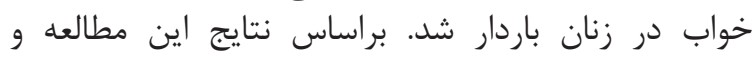

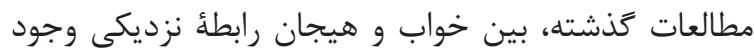

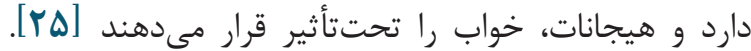

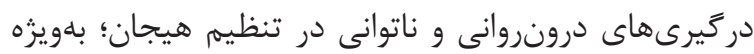

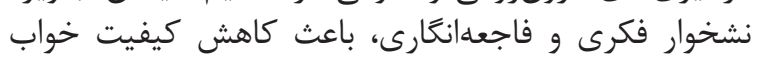

مىشود [F].

افرادى كه در رويارويى با رخدادهاى ناراحت كننده، دركير

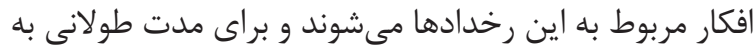

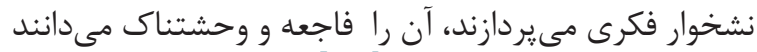

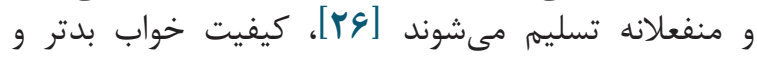

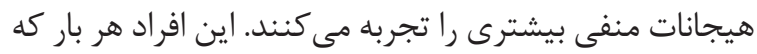

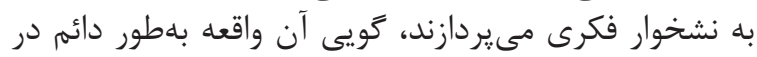

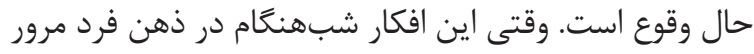

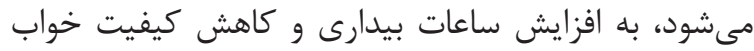

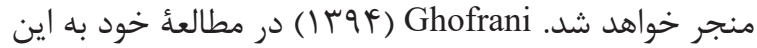
نتيجه رسيد كه فاجعهانگارى كيفيت خواب ران رات تحت تأثير قرار

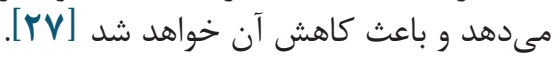
طبق نتايج يزوهش، افرادى كه خود را در وقوع حوادث 
نشخوار فكرى، فاجعه يندارى و سرزنش ديكران و فعاليت زياد

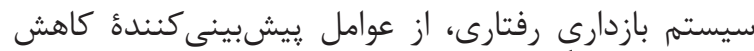

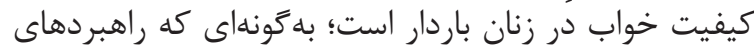

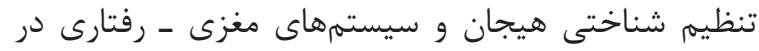

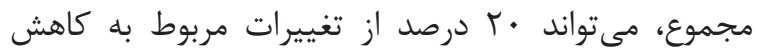
كيفيت خواب را ييشبينى كند.

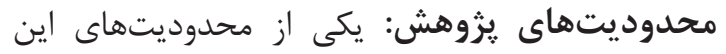

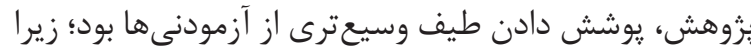

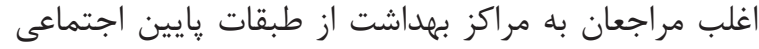

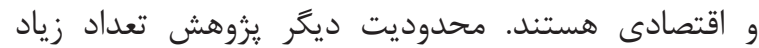

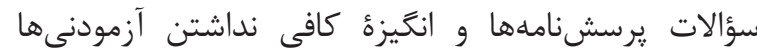

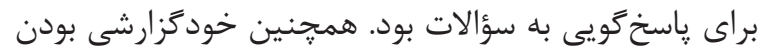

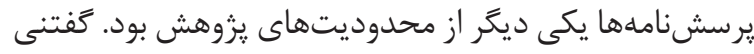

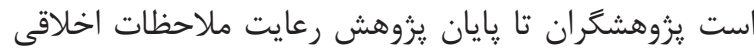

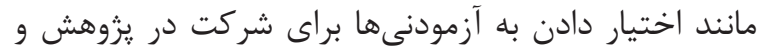

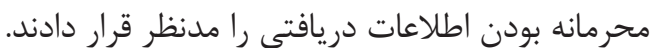

\section{سياسگزارى}

نويسند

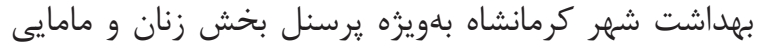

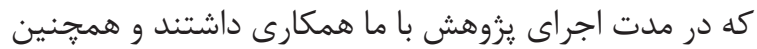

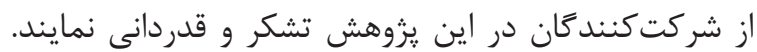

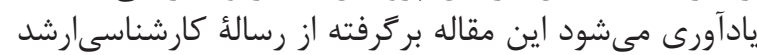

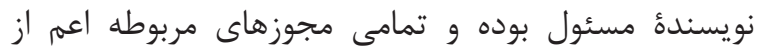

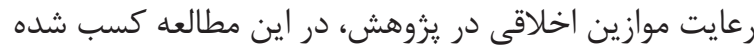

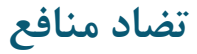

در اين مطالعه تضاد منافع وجود ندارد.
اين يافته با نتايج يزوهش Gharashi ناهمخوان است؛ در

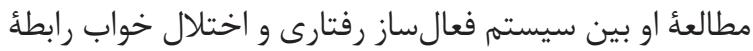

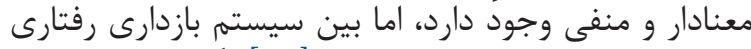

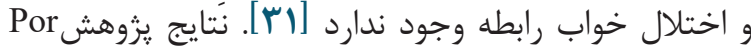
Mirzamani Bafghi g rezaye Tajrishi

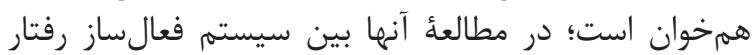

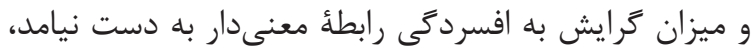

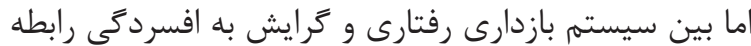

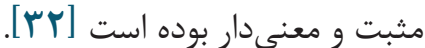

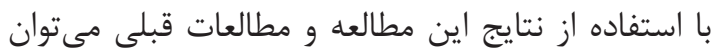

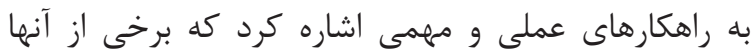

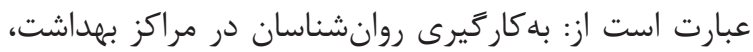

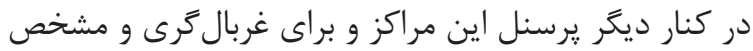

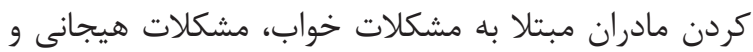

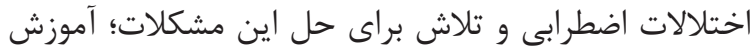

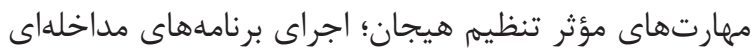

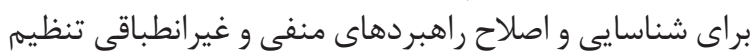

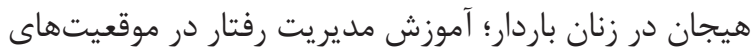

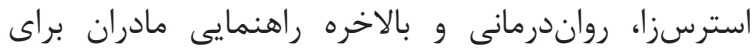
دريافت خدمات روانشناختى.

ييشنهاد مىشود در مطالعات آينده از روشهاى عينىتر

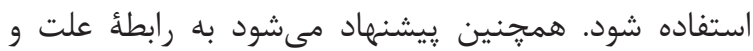

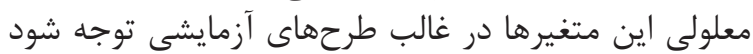

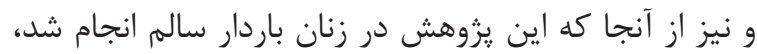

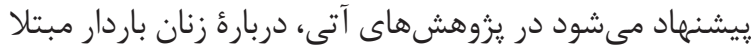

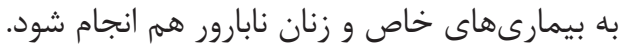

$$
\text { نتيجه كيرى }
$$

\section{References}

1. Moline M, Broch L, Zak R. Sleep problems across the life cycle in women .Curr Treat Options Neurol Jul 2004;6(4):319-30. https://doi. org/10.1007/s11940-004-0031-6

2. Mckee MD, Cunningham M, Jankowski KR, Zayas L. Health-related functional status in pregnancy:relationship to depression and social support in a multi-ethnic population. Obstet Gynecol. 2001; 97(6): 988-93. PMID: 11384708

3. Dogan O, Ertekin S, Dogan S. Sleep quality in hospitalized patients. J Clin Nurs 2005;14(1):107-13. https://doi.org/10.1111/ j.1365-2702.2004.01011.x PMID:15656855

4. Guastella A, Moulds M. The impact of rumination on slepp quality following a stressful life event, personality and individual difference 2007; 42: 1151-1162.

5. Dzaja A, Arber S, Hislop J, Kerkhofs M, Kopp C, Pollmacher T, et al. Women's sleep in health and disease.J Psychiatr Res 2005; 39(1): 55-76. https://doi.org/10.1016/j.jpsychires.2004.05.008 PMid:15504424

6. Lopes EA, Carvalho LB, Seguro PB, Mat$\operatorname{tar}$ R, SilvaAB, Prado LB, et al. Sleep disorders in pregnancy.Arquivos Neuro-Psiquiatr 2004;62:217-21. https://doi.org/10.1590/S0004282X2004000200005

7. Santiago JR, Nolledo MS, Kinzler W, Santiago TV. Sleep and sleep disorders in pregnancy. Annals internal Medicine 2001; 134(5): 396408. https://doi.org/10.7326/0003-4819-134-5200103060-00012

8. Chang JJ, Pien GW, Duntley SP, Macones GA. Sleep deprivation during pregnancy and maternal and fetal outcomes: is there a relationship? Sleep Med Rev. 2010;14(2):107-14. https://doi.org/10.1016/j.smrv.2009.05.001 PMID:19625199

9. Thompson RA. Emotion regulation: a theme in search of definition. Monogr Soc Res 
Child Dev. 1994;59(2-3):25-52.https://doi. org/10.2307/1166137 PMID:7984164

10. Garnefski N, Kraaij V, Spinhoven P. Manual for the use of the cognitive emotion regulation questionnaire.Leiderorp 2002;30:1311-1327.

11. Szczygiel D, Buczny J, Bazinska R. Emotion regulation and emotional information processing: The moderating effect of emotional awareness. Press indiv differ 2012; 52(3): 433-7. https://doi. org/10.1016/j.paid.2011.11.005

12. Samani S,Sadeghi L. The adequacy of psychometric measures of cognitive emotion regulation strategies questionnaire.J psychomodel method 2010; 1(1):51-62

13. Garnefski N, Kraaij V. Relationships between cognitive emotion regulation strategies and deppresive symptoms: A comparative study of five specific samples.Personality and Individual Differences 2006; 40: 1659-1669. https://doi. org/10.1016/j.paid.2005.12.009

14. Gray JA. Personality 7 individual diffrences.A natural science approach.New yourk picnum press 1982.

15. Gray JA. Amodel of the limbic system and basal ganglia:Applications to anxiety and schizophrenia. In Gazzaniga M,(Eds), the cognitive neuroscience 1991;1165-1176.

16. Gray JA. Brain systems that mediate both emotion and cognition.special issue:Development of relationship between emotion and cognition. Cog Emo 1990;(4):269-288.

17. Gray JApt, McNaughton N.The neuropsychology of anxiety:Reprise. In hope DA.(Eds),Nebraska symposium on motivation:Perspective on anxiety,panic,and fear.1996; 43:61-134.

18. Gray JA, McNaughton N.The neuropsychology of anxiety.AN enquiry into the functions of the septohippocampal system.New york:Oxford university press 2000 .

19. Corr PJ. Reinforcement sensitivity theory and personality.Neuroscience and biobehavioral reviews 2004;(28):317-332. https://doi.org/10.1016/j. neubiorev.2004.01.005 PMID: 15225974

20. Buysse DJ, Reynolds CF, Monk TH,Bermaner SR, Kupfer DJ. The Pittsburgh Sleep Quality Index: a new instrument for psychiatric practice and research. Psychiatry Res 1989; 28(2): 193-213. https://doi.org/10.1016/0165-1781(89)90047-4

21. Heydari A, Ehteshamzadeh P, Marashi M.The relationship between insomnia severity,sleep quality, sleepness and impaired mental health and academic performance of girls.J woman and culture 2010;1(4) 65-76
צ Tr. بررسى رابطة كيفيت خواب با راهبردهاى تنظيم شناختى ...

22. Aminabadi Z, Dehghani M, Khodapanahi MK. Factor structure and validity of cognitive emotion regulation questionnaire.Jbehavioral sciences 2011;5(4) 21-22.

23. Carver CS, White TL. Bhavioral inhibition,behavioral activation and effective responses to impending reward and punishment.the BIS/BAS scale.J Personality and Social psychology 1994;67(2):319-33. https:// doi.org/10.1037/0022-3514.67.2.319

24. Abdolahi Majarshin R.The relathionship between inhibition and activation behavioral systems with memory bias in depressed patients.[master thesis].Tabriz university 2006; 57-88

25. Kahn M, Sheppes G, Sadeh A. Sleep and emotions: Bidirectional links and underlying mechanisms. J international of Psychophysiology 2013;89(2) 218-228.

26. Watson w. Few Employers Addressing Workplace Stress, Watson Wyatt Surveys Find. J Press elease 2005;(14):298-306.

27. Ghofrani S.Examines the role of cognitive emotion regulation variables interpersonal problems and sleep quality among parents of middle school students.[master thesis].Iran;Islamic Azad university of Marvdasht;2015

28. Yosefi F.Investigate the relationship between cognitive emotion regulation strategies withderession and anxiety in students talent. Quarterly periodical of research of exceptional children 2006;6(4) :871-892

29. Sutter-Dallay AL,Giaconne,Marcesche V,Glatigny-Dallay E,Verdoul H.Women with anxiety disorders during pregnancy are at incesed risk of intense potantal depressive systems:a prospective survey of the MATAUTD cohort.Europen psychiatry 2004;19(8):459-463. https://doi.org/10.1016/j.eurpsy.2004.09.025 PMID:15589703

30. Jokar E, Rahmati A.The effectivenss of stress management on womens sleep quality.J of mental health principles 2015;2(17):62-66

31. Gharashi Y.The relationship between brain behavioral systems and deppressive symtomps with symtomps of sleep disorders[Master thesis].Iran. faculty of education and psychology Tabriz university;2015.

32. Por Rezaye Tajrishi M, Mirzamani Bafghi M.The relationship between brain-behavioral systems,social support and depression.J social walfar qurterly 2007;7(26):223-246 